\title{
TUBERCULOSIS
}

\section{Epidemiology of tuberculosis on Gran Canaria: a 4 year population study using traditional and molecular approaches}

\author{
M J Pena, J A Caminero, M I Campos-Herrero, J C Rodríguez-Gallego, \\ M I García-Laorden, P Cabrera, M J Torres, B Lafarga, F Rodríguez de Castro, \\ S Samper, F Cañas, D A Enarson, C Martín
}

Thorax 2003;58:618-622

See end of article for authors' affiliations

\section{Correspondence to:} Dr J A Caminero, Service of Neumology, University General Hospital "Dr

Negrín", Barranco de la Ballena s/n, 35020 Las Palmas de Gran Canaria, Spain; jcaminer@separ.es

Revised version received 5 February 2003 Accepted for publication 21 March 2003

\begin{abstract}
Background: In recent years several population based studies using restriction fragment length polymorphism (RFLP) analysis have shown a higher rate of recent transmission of tuberculosis than previously thought. This study was undertaken to determine the transmission patterns of tuberculosis and the potential causes of recent transmission on the island of Gran Canaria (Spain).

Methods: The strains of all patients diagnosed with tuberculosis confirmed by culture between 1 January 1993 and 31 December 1996 were typed by RFLP using the insertion sequence IS61 10. A cluster was defined as two or more isolates with an identical RFLP pattern. Epidemiological linkage through contact tracing was investigated.

Results: Of the total of 719 patients, 153 (21.3\%) were excluded because there was inadequate bacterial DNA for genotyping ( $n=129)$ or the isolates of Mycobacterium tuberculosis had less than five copies of IS6 $110(n=24)$. The isolates from 409 patients (72.3\%) were grouped into 78 different clusters with an estimated $58.5 \%$ of the cases being due to recent transmission. Young age was the only significant predictor of clustering. Only in $147(35.9 \%)$ of the 409 patients belonging to a cluster could an epidemiological link be found. 111 patients (19.6\%) were identified as having had previous contact with a tuberculosis patient and 81 of them (72.9\%) belonged to a cluster. The three largest clusters included 75, 49 and 20 patients, respectively.

Conclusion: Recent transmission is frequent among patients with tuberculosis on Gran Canaria and could be associated with certain aspects of control measures. Some of the clusters described in the study could be due to the prevalence of particular strains of $M$ tuberculosis on the island.
\end{abstract}

$\mathrm{T}$ he decline in tuberculosis (TB) in industrialised countries towards the end of the 19th century was due, at least in part, to the improvement in socioeconomic conditions. In these countries the decreasing prevalence of the disease was accelerated by the introduction of effective drugs. Treatment was able not only to cure the patients, but also significantly to reduce the spread of the disease within the community, which was also an important goal from the perspective of public health. Consequently, in those countries where adequate treatment was systematically employed, the risk of infection of TB declined progressively, in parallel with the decline in numbers of the most contagious cases.

Exogenous infection by Mycobacterium tuberculosis was thought to be very common in the pre-antibiotic era, ${ }^{1}$ but from the turn of the 20th century it gradually decreased to the extent that, in the last two decades, it was thought to be very uncommon. ${ }^{2}$ Moreover, it has been assumed that, in industrialised countries, $90 \%$ of cases of TB were due to endogenous reactivation of a previous infection by $M$ tuberculosis. ${ }^{2}$ Today this belief is subject to more rigorous questioning in the light of the new molecular methods for differentiation of $M$ tuberculosis strains.

The most common and useful method for typing $M$ tuberculosis strains is by restriction fragment length polymorphism associated with the insertion sequence IS6110 (RFLPIS61 10). ${ }^{3}$ Population based molecular epidemiological studies using RFLP-IS6110 have found percentages of clustered strains (thought to indicate recent transmission) of more than $30 \%$, even in countries where the incidence is low such as the US, ${ }^{45}$ Denmark, ${ }^{67}$ and the Netherlands. ${ }^{8}$ These findings raise the question whether recent transmission is more common than was previously thought..$^{4-6}$ On the other hand, the percentage of clustering was similar to that found in studies carried out in developing countries, ${ }^{9-15}$ where it was thought that the high rate of TB in the community was due to a high rate of recent infection or of reinfection.

Over a 4 year period we have carried out a population based study using conventional and molecular epidemiological methods in a relatively closed population on the island of Gran Canaria (where the TB control programme shows cure rates of $>90 \%{ }^{16}$ ) to determine the transmission patterns of TB and to clarify the origin of recent transmissions.

\section{METHODS}

The island of Gran Canaria in Spain has a population of 713768 , nearly half of whom live in the city of Las Palmas. All people have access to the public health service without charge, including immigrants and the homeless.

The study group consisted of all patients with TB confirmed by culture between 1 January 1993 and 31 December 1996 in either of the two referral hospitals on the island (Hospital Insular (HI) and Hospital de Gran Canaria "Dr Negrín" (HN)).

Medical records were systematically reviewed for demographic data (age, sex, race, country of birth), risk factors for TB (homeless, tobacco and alcohol abusers, intravenous drug addiction, and contact with tuberculous patients), and clinical and microbiological characteristics of TB. HIV tests were performed on all patients accompanied by pre- and post-test counselling.

Isolates of $M$ tuberculosis from every patient included in the study were stored at $-40^{\circ} \mathrm{C}$ in Dubos liquid media before being 


\begin{tabular}{|c|c|c|c|c|}
\hline & \multirow[b]{2}{*}{ Total } & \multicolumn{2}{|c|}{ Included } & \multirow[b]{2}{*}{$\operatorname{RR}(95 \% \mathrm{Cl})$} \\
\hline & & Yes & No & \\
\hline \multicolumn{5}{|l|}{ Sex } \\
\hline Male & 497 & 393 & 104 & $1.07(0.72$ to 1.51$)$ \\
\hline Female & 222 & 173 & 49 & \\
\hline \multicolumn{5}{|l|}{ Age (years) } \\
\hline$<35$ & 303 & 246 & 57 & 0.79 (0.54 to 1.16$)$ \\
\hline$>35$ & 349 & 270 & 79 & \\
\hline \multicolumn{5}{|l|}{ Disease } \\
\hline Pulmonary & 643 & 508 & 135 & 1.16 (0.66 to 2.04 ) \\
\hline Extrapulmonary & 76 & 58 & 18 & \\
\hline \multicolumn{5}{|l|}{ Smear } \\
\hline Positive & 365 & 291 & 74 & $1.13(0.79$ to 1.62$)$ \\
\hline Negative & 354 & 275 & 79 & \\
\hline \multicolumn{5}{|l|}{ TB contact } \\
\hline Yes & 118 & 111 & 7 & $0.96(0.36$ to 2.52$)$ \\
\hline No & 326 & 310 & 16 & \\
\hline \multicolumn{5}{|l|}{ HIV } \\
\hline Positive & 118 & 88 & 30 & 0.75 (0.47 to 1.19 ) \\
\hline Negative & 601 & 478 & 123 & \\
\hline \multicolumn{5}{|l|}{ Smoking } \\
\hline Yes & 384 & 332 & 52 & 3.67 (2.49 to 5.43 ) \\
\hline No & 249 & 158 & 91 & \\
\hline \multicolumn{5}{|l|}{ Alcohol abuse } \\
\hline Yes & 195 & 161 & 34 & 1.56 (1.02 to 2.40$)$ \\
\hline No & 438 & 329 & 109 & \\
\hline \multicolumn{5}{|l|}{ IVDA } \\
\hline Yes & 92 & 72 & 20 & 0.96 (0.56 to 1.64$)$ \\
\hline No & 627 & 494 & 133 & \\
\hline \multicolumn{5}{|l|}{ Homeless } \\
\hline Yes & 35 & 32 & 3 & $2.96(0.90$ to 9.78$)$ \\
\hline No & 684 & 534 & 150 & \\
\hline \multicolumn{5}{|l|}{ Prison } \\
\hline Yes & 44 & 40 & 4 & 2.83 (0.99 to 8.04$)$ \\
\hline No & 675 & 526 & 149 & \\
\hline \multicolumn{5}{|l|}{ Immigrant } \\
\hline Yes & 48 & 38 & 10 & $1.02(0.50$ to 2.11$)$ \\
\hline No & 671 & 528 & 143 & \\
\hline
\end{tabular}

RR=relative risk for having RFLP analysis; $95 \% \mathrm{Cl}=$ confidence interval; IVDA=intravenous drug addict.

subcultured on Lowenstein-Jensen media and incubated at $37^{\circ} \mathrm{C}$ in an aerobic atmosphere with $5-10 \% \mathrm{CO}_{2}$. An isolate from each of the patients was genotyped with RFLP-IS6110 85 strains from the period 1991-2 (32.5\% of all isolates in that period) were also typed.

Fingerprint patterns of the isolates were analysed with the Gel Compare 4.0 (Applied Math, Kortijk, Belgium) software program and subsequently by visual examination of the cluster patterns. A cluster of $M$ tuberculosis isolates was defined as two or more isolates revealing an identical number and location of IS6110 when five or more copies of IS6110 were present. ${ }^{6917}$ We assumed that one patient from each cluster corresponded to the index case origin of the infection, ${ }^{45}{ }^{11}$ so that the number of patients in a cluster is equal to $n-1$. Patients with non-matching RFLP-IS6110 were considered non-clustered. Following RFLP-IS6110 analysis, the data for all patients included in the clusters were further reviewed to look for evidence of epidemiological linkage.

Data were analysed using computer software (EPI INFO 6 and SPSS 7.0 statistical programs). Student's $t$ test and $\chi^{2}$ test were used to assess univariate risk factors for being in a cluster and also to compare patients who were genotyped by RFLP-IS6110 with those in whom this study could not be performed. They were then included in multivariate logistic regression model analysis. Significance was defined as $\mathrm{p}<0.05$. Relative risks were calculated from regression estimates based on the probability test for the likelihood ratio statistic; $95 \%$ confidence intervals were based on the estimated variance of the regression coefficients.
Table 2 Number and size of every cluster found in Gran Canaria

\begin{tabular}{lll}
\hline $\begin{array}{l}\text { Cluster size } \\
\text { (no of patients) }\end{array}$ & $\begin{array}{l}\text { No of } \\
\text { clusters }\end{array}$ & $\begin{array}{l}\text { Total no of } \\
\text { patients }\end{array}$ \\
\hline 2 & 40 & 80 \\
3 & 14 & 42 \\
4 & 7 & 28 \\
5 & 4 & 20 \\
6 & 2 & 12 \\
7 & 2 & 14 \\
8 & 2 & 16 \\
10 & 1 & 10 \\
12 & 1 & 12 \\
15 & 1 & 15 \\
16 & 1 & 16 \\
20 & 1 & 20 \\
49 & 1 & 49 \\
75 & 1 & 75 \\
Total & 78 & 409 \\
\hline & & \\
\hline
\end{tabular}

\section{RESULTS}

On the island of Gran Canaria 231 cases of TB were diagnosed ( 214 confirmed by culture) in 1993 (incidence rate 32.4 per 100000 inhabitants), 210 ( 185 confirmed by culture) in 1994 (incidence rate 29.4), 176 ( 142 confirmed by culture) in 1995 (incidence rate 24.6), and 198 (178 confirmed by culture) in 1996 (incidence rate 27.7). A total of 719 culture positive isolates were therefore obtained during the 4 year period of the study ( 527 in NH and 192 in HI). In 129 of these ( 75 in HN and 54 in HI) subcultures of $M$ tuberculosis were not available for DNA fingerprinting (17.9\%). Following RFLP-IS6110 evaluation of the remaining 590 patients, 24 isolates were excluded because they had fewer than five copies of IS6110. The final cases studied thus consisted of 566 patients (437 in HN and 129 in HI): 179 in 1993, 148 in 1994, 110 in 1995, and 129 in 1996; 393 were male and the mean (SD) age was 40.3 (17.4) years (range 1-87). Eighty eight patients (15.5\%) were HIV seropositive.

Table 1 shows a comparison of those with and without RFLP-IS6110 analysis. There was no association with sex, age group, HIV status, being an immigrant, site or type of disease, being homeless, having been in prison, being an intravenous drug addict, or having contact with a patient with TB. Subjects more likely to have had RFLP-IS6110 analysis included those who were tobacco smokers and those abusing alcohol. Some cases did not have certain variables recorded on their recordage $(n=67)$, smoking habit $(n=86)$, alcohol abuse $(n=86)$, and TB contact after contact tracing procedure $(n=275)$.

Of the 566 strains typed by RFLP-IS61 10, 409 (72.3\%) were grouped in 78 clusters. The number and size of each cluster is shown in table 2 . The largest cluster consisted of 75 patients, the second 49, and the third 20. Two other clusters contained 16 and 15 patients each. The RFLP-IS6110 patterns of the largest clusters are shown in fig 1. Assuming that one patient from each cluster corresponds to the index case origin of the infection, of the 409 patients associated in clusters 78 might be excluded as cases of recent transmission of TB. The remaining 331 patients $(58.5 \%)$ are therefore those judged to have had recent transmission of TB between 1993 and 1996. Recent transmission was calculated per year and no significant differences were found between years: 131 patients in 31 clusters $(73.2 \%)$ in 1993, 97 in 20 clusters $(65.5 \%)$ in 1994, 79 in 17 clusters $(71.8 \%)$ in 1995 , and 102 in 11 clusters $(79.1 \%)$ in 1996.

To identify risk factors associated with recent transmission, the 409 clustered patients were compared with the 157 patients not clustered. Complete epidemiological information was available for 399 patients (70.5\%). In 167 patients we had some difficulties in obtaining some of the epidemiological 


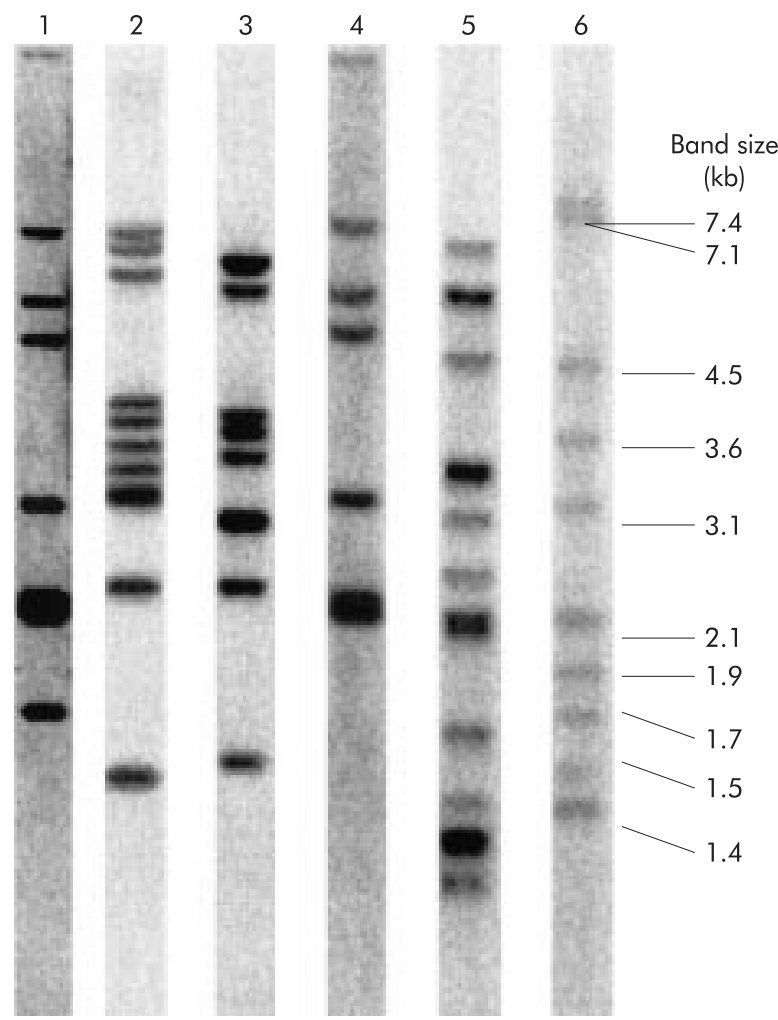

Figure 1 Results of RFLP analysis of $M$ tuberculosis strains isolated from three or more patients.

characteristics, most of them diagnosed in HI. The comparison of clustered and non-clustered patients is shown in table 3. In univariate analysis only age was associated with clustering $(\mathrm{p}=0.02)$, with those less than 45 years of age being significantly more likely to be clustered (relative risk (RR) $1.51,95 \%$ CI 1.01 to 2.25). The association with age disappeared when age was included in a model with other variables in multivariate logistic regression analysis, and none of the other variables was found to be significantly associated with clustering. In spite of the high prevalence of HIV infection in the population with TB $(15.5 \%)$, there was no greater clustering in the HIV positive population than in the HIV negative population.

Additional studies on the 409 patients included in the 78 clusters were carried out, although epidemiological linkage could only be established in 147 (35.9\%). No epidemiological connections were found in patients with a unique RFLPIS61 10 pattern. The two largest clusters differed from all other clustered cases in being more likely to have been in prison (RR $2.79,95 \%$ CI 1.44 to 5.38 ), to be intravenous drug addicts (RR $2.77,95 \%$ CI 1.68 to 4.55 ), to be immigrants (RR $2.05,95 \%$ CI 1.01 to 4.18 ), and to be HIV positive (RR $1.77,95 \%$ CI 1.15 to 2.73). The largest cluster of 75 patients (cluster 1) was the basis of a previous publication ${ }^{18}$ and differed from the second largest in being immigrants, intravenous drug addicts (RR 8.36, 95\% CI 2.08 to 33.53 ), and in being HIV positive (RR 3.00, $95 \%$ CI 1.23 to 7.30 ). An epidemiological linkage was found in $68 \%$ of the cases.

Cluster 2 comprised 49 patients with an identical RFLPIS6110 pattern (seven bands of IS6110, fig 1). Seven patients were diagnosed in 1993, nine in 1994, 13 in 1995, and 16 in 1996. In $63 \%$ of the patients it was possible to establish an epidemiological linkage. This cluster did not include immigrants and most of the patients were natives of the island. The cluster was spread throughout five different areas of the island, most of them of a low socioeconomic level. This RFLPIS6110 pattern was also found in three of 85 patients $(3.5 \%)$ diagnosed during the 1991-2 period, suggesting that these strains were in the island before 1992. The index case of the cluster was probably an alcoholic HIV negative patient from Las Palmas who adhered to a directly observed therapy programme ${ }^{19}$ and was cured 6 months later. Epidemiological connections were found in two patients related to the index case. They disseminated the disease to another two areas of the city where the cluster was found.

Cluster 3 included 20 patients with an RFLP pattern of 10 bands of IS6110 (fig 1). Ten strains were isolated in 1993, four in 1994, two in 1995, and four in 1996. An epidemiological linkage could be established in $70 \%$ of the patients, most of them belonging to a cluster found in Gáldar (a city on the north coast of the island) and in Las Palmas. This RFLP-IS6110 pattern was found in two of 85 patients $(2.3 \%)$ studied during the years 1991 and 1992, suggesting that the strains were in the island before 1992. The index case of the cluster might have been an alcoholic (HIV negative) patient with poor compliance with treatment. Originally from Gáldar, he lived for some periods in Las Palmas where most of the other patients of this cluster were found.

There was another cluster containing 16 patients which included two homeless people. No epidemiological connection was established between these patients. The specific RFLPIS61 10 pattern was present in three strains out of the 85 studied from 1991 to 1992 . The cluster containing 15 patients included four HIV positive patients (26.7\%) and one who was homeless. An epidemiological linkage was found in $13.3 \%$ of the patients and the RFLP-IS6110 pattern was shared by 14 isolates from the 85 patients (16.5\%) in the period 1991-2.

Of the 566 patients included in the study, only 111 (19.6\%) identified previous contact with other TB patients. Eighty one $(72.9 \%)$ belonged to one of the 78 clusters studied: 13 to cluster 1,15 to cluster 2 , four were found in a cluster of 20 people, and two in a cluster of 15 patients. Forty clusters consisting of two patients each were further studied by conventional contact tracing. An epidemiological linkage could be found in only three $(7.5 \%)$ of them. There was no association between the size of the cluster and the probability of having a record of TB contact.

\section{DISCUSSION}

The development of new molecular methods to genetically differentiate $M$ tuberculosis strains provides a useful epidemiological tool. The first described method for differentiating $M$ tuberculosis isolates used mycobacteriophages ${ }^{20}{ }^{21}$ or enzymatic characterisation..$^{22}$ Although useful for the study of specific outbreaks, ${ }^{20-22}$ these techniques had very low discriminatory power. In the last 10 years DNA fingerprinting of $M$ tuberculosis isolates has increasingly been used in epidemiological studies. These techniques primarily involve RFLP analysis using the insertion sequence IS6110. The RFLP-IS6110 methods have proved to be useful for investigating TB outbreaks, ${ }^{23}$ and their discriminatory power has raised some questions on the interpretation of TB transmission based on classical epidemiological studies. ${ }^{2}$ For example, molecular epidemiology studies in the US have estimated a recent transmission rate of more than $30 \%$ of TB cases,${ }^{45}$ considerably higher than the generally accepted figure of $10 \%{ }^{2}$ The percentage of patients found in clusters was 35\% in Amsterdam, ${ }^{8} 25 \%$ in Paris, ${ }^{24}$ and less than $20 \%$ in Zurich. ${ }^{25}$ More surprising are the results in Denmark ${ }^{7}$ where $49 \%$ of patients were associated with a cluster, a figure that reached $79 \%$ in Greenland. This contrasts with Norway ${ }^{26}$ with a substantially lower proportion of cases in clusters. These percentages are similar to those described for developing countries where the epidemiology of $\mathrm{TB}$ is not under control. ${ }^{9-15}$ In South Africa $30 \%$ of cases are clustered, ${ }^{9}$ a rate lower than the $39.2 \%$ found in Ethiopia ${ }^{10}$ or the $41 \%$ in Tunisia, ${ }^{11}$ with a 10 times lower incidence of TB than in Ethiopia. On the other hand, in developing countries some studies 
Table 3 Characteristics of patients associated with clustering of RFLP

\begin{tabular}{|c|c|c|c|c|}
\hline & \multicolumn{2}{|l|}{ Cluster } & \multirow{2}{*}{$\begin{array}{l}\text { Univariate analysis } \\
\operatorname{RR}(95 \% \mathrm{CI})\end{array}$} & \multirow{2}{*}{$\begin{array}{l}\text { Multivariate analysis } \\
\operatorname{RR}(95 \% \mathrm{Cl})\end{array}$} \\
\hline & Yes (\%) & No & & \\
\hline \multicolumn{5}{|l|}{ Sex } \\
\hline Male & 279 (70.9) & 114 & $0.80(0.53$ to 1.21$)$ & $0.82(0.48$ to 1.40$)$ \\
\hline Female & $130(75.1)$ & 43 & & \\
\hline \multicolumn{5}{|l|}{ Age (years) } \\
\hline$<45$ & $255(75.0)$ & 85 & 1.51 (1.01 to 2.25$)$ & $1.50(0.91$ to 2.46$)$ \\
\hline$>45$ & $117(66.4)$ & 59 & & \\
\hline \multicolumn{5}{|l|}{ Disease } \\
\hline Pulmonary & 371 (72.9) & 138 & 1.66 (0.92 to 2.99 ) & $2.00(0.94$ to 4.25$)$ \\
\hline Extrapulmonary & $38(65.5)$ & 20 & & \\
\hline \multicolumn{5}{|l|}{ Smear (RS) } \\
\hline Positive & 204 (74.2) & 71 & 0.96 (0.68 to 1.41$)$ & 0.89 (0.53 to 1.49 ) \\
\hline Negative & 205 (70.4) & 86 & & \\
\hline \multicolumn{5}{|l|}{ TB contact } \\
\hline Yes & $81(73,0)$ & 30 & 1.11 (0.68 to 1.81$)$ & $1.00(0.59$ to 1.69$)$ \\
\hline No & $220(70.9)$ & 90 & & \\
\hline \multicolumn{5}{|l|}{ HIV } \\
\hline Positive & $67(76.1)$ & 21 & $1.26(0.74$ to 2.15$)$ & 1.12 (0.54 to 2.32 ) \\
\hline Negative & $342(71.6)$ & 136 & & \\
\hline \multicolumn{5}{|l|}{ Smoking } \\
\hline Yes & 239 (71.9) & 93 & 0.99 (0.65 to 1.51$)$ & $1.00(0.58$ to 1.73$)$ \\
\hline No & 114 (72.1) & 44 & & \\
\hline \multicolumn{5}{|l|}{ Alcohol abuse } \\
\hline Yes & 117 (72.7) & 44 & 1.04 (0.68 to 1.59 ) & $0.99(0.57$ to 1.72$)$ \\
\hline No & $236(71.7)$ & 93 & & \\
\hline \multicolumn{5}{|l|}{ IVDA } \\
\hline Yes & $54(75.0)$ & 18 & 1.17 (0.66 to 2.07$)$ & 0.91 (0.40 to 2.07$)$ \\
\hline No & $355(71.9)$ & 139 & & \\
\hline \multicolumn{5}{|l|}{ Homeless } \\
\hline Yes & $25(78.1)$ & 7 & 1.39 (0.59 to 3.29$)$ & $1.27(0.45$ to 3.58$)$ \\
\hline No & $384(71.9)$ & 150 & & \\
\hline \multicolumn{5}{|l|}{ Prison } \\
\hline Yes & $33(82.5)$ & 7 & 1.88 (0.81 to 4.34$)$ & 2.31 (0.49 to 10.89$)$ \\
\hline No & $376(71.5)$ & 150 & & \\
\hline \multicolumn{5}{|l|}{ Immigrant } \\
\hline Yes & $28(73.7)$ & 10 & $1.08(0.51$ to 2.27$)$ & 2.10 (0.58 to 7.54$)$ \\
\hline No & $381(72.2)$ & 147 & & \\
\hline
\end{tabular}

have shown that recent transmission is lower than in industrialised countries. ${ }^{12}{ }^{13}$ These discrepancies between molecular and traditional epidemiological approaches require detailed analysis and evaluation. This study of the total population living on an island with a moderate incidence of TB may help to clarify this controversy.

Our study was performed over one of the longest periods (4 years) described to date, ${ }^{45} 725$ 27-29 together with the study by Bauer $e a^{6}{ }^{6}$ in Denmark and Greenland. In our study $72.3 \%$ of 566 patients were grouped into 78 clusters. Even if we eliminate one patient per cluster (index case), ${ }^{4911}$ the clustered patients represent $58.5 \%$ of the total number of patients with TB during the period 1993-6 - that is, the percentage of TB due to recent transmission-which is similar if we calculate it per year. This is the highest rate recorded to date, ${ }^{45-1530}$ including countries with a higher incidence of $\mathrm{TB},{ }^{9}{ }^{10}$ and is similar to that reported by Bauer $e a^{6}{ }^{6}$ in the study carried out in Greenland.

The high rate of recent transmission found in Gran Canaria is unexpected since there is free and universal access to the public health service and excellent detection and cure rates, ${ }^{16}$ even in marginal population areas. ${ }^{19}$ However, we believe that our high percentage of clustering does not provide absolute evidence of recent transmission, as has been suggested by previous studies. ${ }^{17} 2729$ This is supported by the following: (1) our total number of TB patients ${ }^{16}$ is against a high rate of recent transmission; (2) the good detection and cure rates in the last years ${ }^{16} ;(3)$ only in $35.9 \%$ of the patients associated with clusters was it possible to establish an epidemiological linkage, which is similar to other studies ${ }^{4} 9^{25} 27-29$; and (4) only in $7.5 \%$ of the patients belonging to clusters of two patients was it possible to show an epidemiological connection.

Molecular methods have been convincingly shown to be very useful in outbreak studies (including those concerning multidrug resistant strain ${ }^{23}$ ) as well as in the evaluation of laboratory cross contamination, ${ }^{31}$ contact tracing studies, ${ }^{32}$ and in the differentiation between exogenous reinfections and endogenous reactivation. ${ }^{33}$ However, in population based studies the number of variables is higher and the results of clustering should be interpreted with caution. When only conventional epidemiological contact tracing is considered, it is possible to underestimate cases of recent transmission, particularly whether the contact is sporadic or the tracing is nonexhaustive. ${ }^{28}$ On the other hand, if all the cases in a cluster, as defined by RFLP-IS6110, are assumed to result from recent transmission, then this figure could be overestimated. Yang et $a l^{27}$ compared results from different US states and found that some $M$ tuberculosis strains had an identical RFLP-IS6110 pattern in different states without evidence of recent transmission. This could explain the high rate of clustering found in our study, as in others carried out on islands. ${ }^{6}$ In these closer communities it is possible to find some highly prevalent $M$ tuberculosis strains, which may explain the discrepancies between the results obtained using conventional epidemiological methods and RFLP-IS6110. ${ }^{49} 917252829$ It may be necessary to use complementary typing methods to assess recent transmission reliably. ${ }^{35}$

In our study the larger clusters had a considerable impact, with the largest comprising $13.2 \%$ of the patients and the second largest $8.6 \%$. This suggests the presence of prevalent 
microorganisms with a high capacity for infecting and developing the disease, as has been previously suggested. ${ }^{4-6} 36$ Cluster 1 (75 patients) is one of the largest reported so far. ${ }^{4}{ }^{7-10} 2430$ The only comparable clusters have been described in Greenland, ${ }^{6}$ a region that could have been influenced by similar factors to those in this study. The impact of this single strain on the epidemiology of TB on our island may be important. This highly prevalent strain entered Gran Canaria in 1993 from an African immigrant. It appeared to disseminate among susceptible populations, including HIV positive subjects, drug abusers and immigrants. ${ }^{18}$ As long as an epidemiological linkage was demonstrated in $68 \%$ of the cases, it could be assumed that this particular cluster could be related to recent transmission. Transmission of particularly infectious strains could therefore be possible after a sporadic contact, as has previously been suggested. ${ }^{28}$ In other clusters, however, the presence of prevalent strains on the island for a long time makes it difficult to assume that these are related to recent transmission..$^{37}$

We conclude that the rate of recent transmission in our community is high, which indicates the need to increase control measures, mainly in the immigrant population. To assess compliance with treatment, legal measures may be necessary to help to control the disease. ${ }^{38}$ RFLP-IS6110 is a good method for studying the behaviour of TB in a community, especially as a complement to the classic epidemiological approach and for identifying highly infectious strains.

\section{Authors' affiliations}

M J Pena, M I Campos-Herrero, B Lafarga, Service of Microbiology, University General Hospital "Dr Negrín", Las Palmas de Gran Canaria, Spain

J A Caminero, P Cabrera, F Rodríguez de Castro, Service of Neumology, University General Hospital "Dr Negrín"

J C Rodríguez-Gallego, M I García-Laorden, Service of Immunology, University General Hospital "Dr Negrín"

M J Torres, Research Unit, University General Hospital "Dr Negrín"

J A Caminero, D A Enarson, International Union against Tuberculosis and Lung Disease, Paris, France

S Samper, C Martín, Department of Microbiology, University of Zaragoza, Zaragoza, Spain

F Cañas, Service of Microbiology, Insular Hospital of Gran Canaria, Las Palmas de Gran Canaria, Spain

Supported by Fondo de Investigación Sanitaria grant 96/0676 and the Red-Respira RTIC C03/11, Ministerio de Sanidad y Consumo, Spain.

\section{REFERENCES}

1 Styblo K. Epidemiology of tuberculosis. Jena, East Germany: VEB Gustav Fischer, 1984: 119-20.

2 Anon. The use of preventive therapy for tuberculosis infection in the United States: recommendations of the Advisory Committee for Elimination of Tuberculosis. MMWR 1990;39(RR-8):9-12

3 van Embden JDA, Cave MD, Crawford JT, et al. Strain identification of Mycobacterium tuberculosis by DNA fingerprinting: recommendations for a standarized methodology. J Clin Microbiol 1993;31:406-9.

4 Small PM, Hopewell PC, Singh SP, et al. The epidemiology of tuberculosis in San Francisco. A population-based study using conventional and molecular methods. N Engl J Med 1994;330:1703-9.

5 Alland D, Kalkut GE, Moss AR, et al. Transmission of tuberculosis in New York City. An analysis by DNA fingerprinting and conventional epidemiologic methods. N Engl J Med 1994;330:1710-6.

6 Bauer J, Yang Z, Poulsen L, et al. Results from 5 years of nationwide DNA fingerprinting of Mycobacterium tuberculosis complex isolates in a country with a low incidence of $M$ tuberculosis infection. J Clin Microbio 1998;36:305-8

7 Yang ZH, Haas PEW, Wachmann $\mathrm{CH}$, et al. Molecular epidemiology of tuberculosis in Denmark in 1992. J Clin Microbiol 1995;33:2077-81.

8 Van Deutekom H, Gerritsen JJ, van Soolingen D, et al. A molecular epidemiological approach to studying the transmission of tuberculosis in Amsterdam. Clin Infect Dis 1997;25:1071-7.

9 Warren R, Hauman J, Beyers $N$, et al. Unexpectedly high strain diversity of Mycobacterium tuberculosis in a high-incidence community. S Afr Med J 1996;86:45-9

10 Hermans PWM, Messadi F, Guebrexabher H, et al. Analysis of the population structure of Mycobacterium tuberculosis in Ethiopia, Tunisia, and the Netherlands: usefulness of DNA typing for global tuberculosis epidemiology. J Infect Dis 1995;171:1504-13.
11 Chevrel-Dellagi D, Abderrahman A, Haltiti R, et al. Large-scale DNA fingerprinting of Mycobacterium tuberculosis strains as a tool for epidemiological studies of tuberculosis. J Clin Microbio 1993;31:2446-50.

12 Godfrey-Faussett P, Stoker NG. Aspects of tuberculosis in Africa. Genetic fingerprinting for clues to the pathogenesis of tuberculosis. Trans $R$ Soc Trop Med Hyg 1992;86:472-5.

13 Gómez Marín JE, Rigouts L, Villegas Londoño LE, et al. Restriction fragment length polymorphism (RFLP) analysis and tuberculosis epidemiology. Bull Pan Am Health Organ 1995;29:226-36.

14 Palittapongarnpim P, Luangsook P, Tansuphaswadikul S, et al. Restriction fragment length polymorphism study of Mycobacterium tuberculosis in Thailand using IS61 10 as probe. Int J Tuberc Lung Dis 1997:1:370-6.

15 Yuen LK, Ross BC, Jackson KM, et al. Characterization of Mycobacterium tuberculosis strains from Vietnamese patients by Southern blot hybridization. J Clin Microbiol 1993;31:1615-8.

16 Caminero JA, Díaz F, Rodríguez de Castro F, et al. The epidemiology of tuberculosis in Gran Canaria, Canary Islands, 1988-92: effectiveness of control measures. Tuberc Lung Dis 1995;76:387-93.

17 Van Soolingen D, de Haas PE, Hermans PV, et al. Comparison of various repetitive DNA elements as genetic markers for strain differentiation and epidemiology of Mycobacterium tuberculosis. J Clin Microbiol 1993;31:1987-95.

18 Caminero JA, Pena M, Campos-Herrero Ml, et al. Epidemiological evidence of the spread of a Mycobacterium tuberculosis strain of the Beijing genotype on Gran Canaria island. Am J Respir Crit Care Med 2001;164:1165-70.

19 Caminero JA, Pavón JM, Rodríguez de Castro F, et al. Evaluation of a directly observed six month fully intermittent treatment regimen in patients suspected of poor compliance. Thorax 1996;51:1130-3.

20 Raleigh JW, Wichelhausen R. Exogenous reinfection with Mycobacterium tuberculosis confirmed by phage typing. Am Rev Respir Dis 1973; 108:639-42.

21 Bates JH, Stead W, Rado TA. Phage type of tubercle bacilli isolated from patients with two or more sites of organ involvement. Am Rev Respir Dis 1976;114:353-8.

22 Roman MC, Sicilia M. Preliminary investigation of Mycobacterium tuberculosis biovars. J Clin Microbiol 1984;20:1015-6.

23 Daley CL, Small PM, Schecter GF, et al. An outbreak of tuberculosis with accelerated progression among persons infected with the human immunodeficiency virus. An analysis using restriction-fragment-length polymorphisms. N Engl J Med 1992;326:231-5

24 Gutiérrez MC, Vincent $V$, Aubert $D$, et al. Molecular fingerprinting of Mycobacterium tuberculosis and risk factors for tuberculosis transmission in Paris, France, and surrounding area. J Clin Microbiol 1998;36:486-92

25 Pfyffer GE, Strässle A, Rose N, et al. Transmission of tuberculosis in the metropolitan area of Zurich: a 3 year survey based on a DNA fingerprinting. Eur Respir J 1998;11:804-8.

26 Dahle UR, Sandven P, Heldal E, et al. Molecular epidemiology of Mycobacterium tuberculosis in Norway. J Clin Microbiol 2001;39:1802-7.

27 Yang Z, Bames PF, Chaves F, et al. Diversity of DNA fingerprinting of Mycobacterium tuberculosis isolates in the United States. J Clin Microbio 1998;36:1003-7.

28 Genewein A, Telenti A, Bernasconi C, et al. Molecular approach to identifying route of transmission of tuberculosis in the community. Lancet 1993;342:841-4

29 Braden CR, Templeton GL, Cave MD, et al. Interpretation of restriction fragment length polymorphism analysis of Mycobacterium tuberculosis isolates from a state with a large rural population. J Infect Dis 1997; 175: 1446-52.

30 Samper S, Iglesias $M$, Rabanaque $M$, et al. The molecula epidemiology of tuberculosis in Zaragoza, Spain: a retrospective epidemiological study in 1993. Int J Tuberc Lung Dis 1998;2:281-7.

31 Small PM, McClenny NB, Singh SP, et al. Molecular strain typing of Mycobacterium tuberculosis to confirm cross-contamination in the mycobacteriology laboratory and modification of procedures to minimize occurrence of false-positive cultures. J Clin Microbiol 1993;31:1677-82.

32 Hermans PWM, van Soolingen D, Dale JW, et al. Insertion element IS986 from Mycobacterium tuberculosis: a useful tool for the diagnosis and epidemiology of tuberculosis. J Clin Microbiol 1990;28:2051-8.

33 Small PM, Shafer RW, Hopewell PC, et al. Exogenous reinfection with multidrug-resistant Mycobacterium tuberculosis in patients with advanced HIV infection. N Engl J Med 1993;328: 1 137-44.

34 Caminero JA, Pena M, Campos-Herrero Ml, et al. Exogenous reinfection with tuberculosis on a European Island with a moderate incidence of disease. Am J Respir Crit Care Med 2001:163:717-20.

35 Warren R, Richardson M, Sampson S, et al. Genotyping of Mycobacterium tuberculosis with additional markers enhances accuracy in epidemiologic studies. J Clin Microbiol 1996;34:2219-24.

36 Bifani PJ, Plikaytis BB, Kapur V, et al. Origin and interstate spread of a New York City multidrug-resistant Mycobacterium tuberculosis clone family. JAMA 1996;275:452-7.

37 Van Soolingen D, Hermans PWM. Epidemiology of tuberculosis by DNA fingerprinting. Eur Respir J 1995;8(Suppl 20):649-56s.

38 Campion EW. Liberty and the control of tuberculosis. N Engl J Med $1999 ; 340: 385-6$ 